\title{
Análise de Processamento Distribuído da Operação Hash Join*
}

\author{
Marisa S. Franco, Simone Dominico, Eduardo C. de Almeida, Marco A. Z. Alves \\ ${ }^{1}$ Departamento de Informática - Universidade Federal do Paraná (UFPR) \\ \{masf18, sdominico, eduardo, mazalves\}@inf.ufpr.br
}

\begin{abstract}
Resumo. A Software-defined wide-area networking (SD-WAN) é uma tecnologia que permite (re)programar dispositivos de rede via software. Nesse caso, os dispositivos de rede possuem uma flexibilidade maior na administração, permitindo a programabilidade da rede. Este artigo apresenta uma avaliação do processamento distribuído de operadores hash join de banco de dados em dispositivos de rede, a partir de uma análise baseada em modelo de custo.
\end{abstract}

\section{Introdução}

Quantidades massivas de dados têm sido geradas em taxas crescentes. Paralelamente, a capacidade computacional de coleta, processamento e armazenamento de dados aumentou exponencialmente na última década. Nesse contexto, as pesquisas sobre bancos de dados distribuídos ganharam cada vez mais relevância. O principal foco dos estudos é o custo de comunicação para transferência dos dados que sofrem, principalmente, com a latência de rede. Trata-se de um gargalo importante para melhoria de desempenho de consultas em sistemas de banco de dados nas quais a comunicação entre servidores é exigida, como aquelas nas quais são realizadas operações de junção. Além disso, com a expansão dos serviços na Internet, a tradicional Wide Area Network (WAN) perde espaço para uma nova tecnologia, a SD-WAN. Isso ocorre devido à baixa flexibilidade no uso da WAN tradicional para alguns acessos, como em serviços em cloud. A SD-WAN busca reduzir a complexidade da WAN por meio da virtualização de serviços.

Dessa forma, o roteador possui uma flexibilidade maior na administração, permitindo a programabilidade da rede via softwares desenvolvidos para esta finalidade [Shin et al. 2012]. Assim, elementos como os roteadores assumem uma posição de destaque devido a sua maior flexibilidade. A programabilidade da arquitetura SD-WAN proporciona novas possibilidades para mitigar latências de forma dinâmica, realizando o processamento das consultas nesses dispositivos. Na literatura, estudos do tipo survey tanto sobre operações de join [Yang and Singhal 1997] quanto sobre a tecnologia SD-WAN [Yang et al. 2019] não abordam o processamento de consultas em roteadores. Diante desse cenário, este artigo tem como objetivo avaliar o processamento distribuído de hash join em dispositivos de rede, por meio de uma análise baseada em modelo de custo. A análise realizada contempla a divisão da carga de trabalho de diferentes formas, incluindo o processamento total das consultas no roteador. Dessa forma, avaliou-se o potencial do processamento de dados em dispositivos de rede para ganho de desempenho.

\section{Bancos de Dados Distribuídos e Operação de Join}

Um sistema de banco de dados distribuído é uma coleção de bases de dados locais e remotos conectados através de uma rede de computadores. Utilizando vários computadores

\footnotetext{
*Este trabalho é parcialmente suportado pela CAPES e Instituto Serrapilheira (grant Serra-1709-16621).
} 
chamados de nós, um banco de dados distribuído divide um problema grande em partes menores e menos complexas que são resolvidas de forma distribuída. As arquiteturas distribuídas de banco de dados são baseadas, principalmente, no modelo "cliente-servidor". Nessa arquitetura, o nó cliente envia uma consulta para nós servidores. Esses, por sua vez, respondem com os dados necessários para a consulta. No processamento de consultas distribuídas, a transferência de dados entre os nós de computação acontece por meio de uma rede dedicada ou de Internet. O custo destas consultas está diretamente ligado ao custo da transferência de dados na rede [Kossmann 2000]. Assim, diferentes algoritmos de processamento de consulta concentram-se na geração de planos de consulta que minimizem a quantidade de dados transferidos na rede. As consultas que possuem a operação de join utilizam semi-joins e bloom filter para minimizar a transferência dos dados na rede. Uma operação de join em uma consulta é um modo de recuperar dados de várias tabelas de banco de dados relacionais por meio de combinações lógicas entre as tabelas.

\section{Metodologia e Modelagem Teórica}

Para a avaliação do processamento distribuído de hash join, foram adotados alguns cenários de transmissão de dados baseados em topologia estrela, com dois servidores de banco de dados e um cliente conectados por um roteador. Foi escolhida ainda uma simplificação da consulta (query-10) do benchmark TPC-H [Council 2020], incluindo apenas a operação de join. A consulta foi implementada em $\mathrm{C}^{1}$. Para a criação das tabelas hash, foi utilizada a função FNV-1a [Fowler et al. 2013] - com excelente desempenho em trabalhos anteriores [Estébanez et al. 2014][Scheidt de Cristo et al. 2019]. Foram considerados seis cenários de processamento distribuído de hash join detalhados na Tabela 1.

Tabela 1. Cenários de processamento distribuído de hash join analisados

\begin{tabular}{|l|l|l|}
\hline Armazenamento inicial & Processamento & Cenário \\
\hline \multirow{2}{*}{$\begin{array}{l}\text { Relação maior no servidor 1 e menor } \\
\text { no servidor 2. }\end{array}$} & Relação maior $\rightarrow$ Relação menor. Processamento no servidor 2. \\
\cline { 2 - 3 } & Relação menor $\rightarrow$ Relação maior. Processamento no servidor 1. & 1 \\
\cline { 2 - 3 } $\begin{array}{l}\text { Cada servidor possui uma metade das } \\
\text { duas relações. }\end{array}$ & $\begin{array}{l}\text { Cada servidor faz uma fase de análise. Troca de dados das tabelas hash } \\
\text { entre servidores. Cada servidor processa parte da análise, usando as } \\
\text { duas tabelas hash e sua metade da relação maior. }\end{array}$ & 4 \\
\cline { 2 - 4 } & Todas $\rightarrow$ Roteador. Processamento no roteador. & 5 \\
\hline $\begin{array}{l}\text { Cada servidor possui uma metade das } \\
\text { duas relações, com dados relevantes co- } \\
\text { localizados. }\end{array}$ & Processamento local. & 6 \\
\hline
\end{tabular}

Cada cenário foi avaliado com três cargas de trabalho (1 GB, $10 \mathrm{~GB}$ e $100 \mathrm{~GB})$ e usando três tecnologias de rede (Ethernet $1 \mathrm{~Gb}$, Ethernet $10 \mathrm{~Gb}$ e InfiniBand HDR $12 \times$ ), com comunicação half-duplex e full-duplex. No caso dos cenários 3 e 5, foram avaliadas ainda três velocidades de processamento do roteador: igual às CPUs dos servidores, $5 \% \mathrm{e}$ $10 \%$ mais lentas do que as CPUs. Nos experimentos, foi utilizada uma máquina com dois soquetes, cada um deles com um Intel Xeon Silver 4114 executando o sistema operacional Ubuntu, versão 18.04.01 LTS. Para obtenção dos tempos de execução para o cálculo, executou-se $20 \times$ cada cenário base, combinando as variáveis a seguir: tamanho da carga de trabalho (1 GB, 10 GB e 100 GB de armazenamento), capacidade de rede, paralelismo da rede, velocidade do roteador. Para os resultados, foram usadas as médias dos tempos das fases de construção, análise e impressão dos resultados em cada cenário base. A Tabela 2 descreve os cálculos feitos para os tempos de execução em cada cenário.

\footnotetext{
${ }^{1}$ Hash join: https://github.com/marisasel/hashjoin
} 
Tabela 2. Cálculo de custo utilizado em cada cenário

\begin{tabular}{l}
\hline \multirow{2}{*}{ Cenário } \\
\cline { 2 - 3 }
\end{tabular}

\section{Análise dos Resultados}

A Figura 1 mostra os resultados obtidos com a query-10 do TPC-H por meio do cálculo dos tempos de processamento em cada cenário, detalhados na Tabela 2.

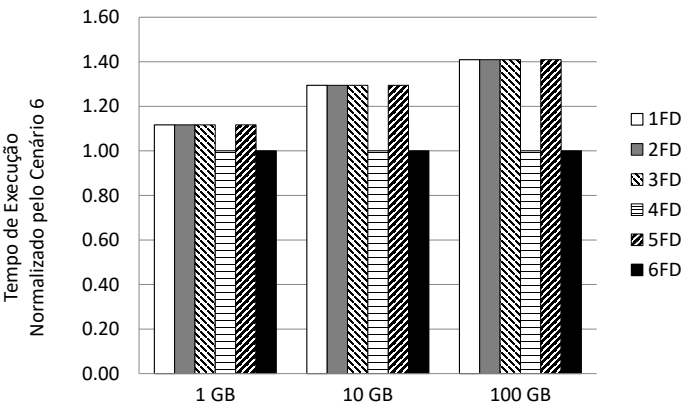

(a) InfiniBand HDR, full-duplex, roteador $100 \%$.

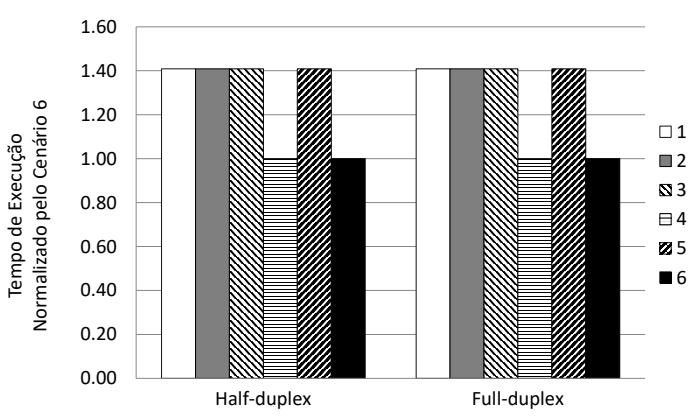

(c) $100 \mathrm{~GB}$, InfiniBand HDR, roteador $100 \%$.

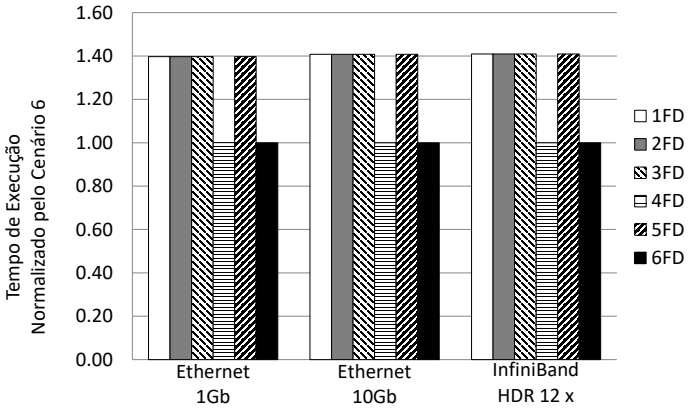

(b) $100 \mathrm{~GB}$, full-duplex, roteador $100 \%$.

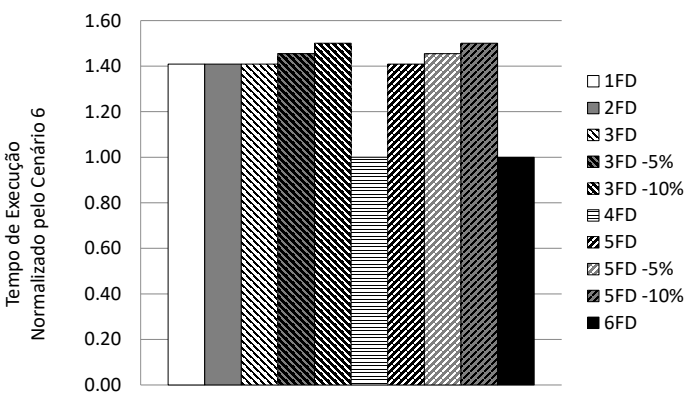

(d) $100 \mathrm{~GB}$, InfiniBand HDR, full-duplex.

Figura 1. Avaliação de desempenho da "Query-10", usando FNV-1a, com os tempos de execução normalizados pelo cenário 6 .

A Figura 1 (a) mostra "melhor cenário": InfiniBand HDR 12×, full-duplex, roteador $100 \%$. Na Figura 1 (b), a combinação dos parâmetros expressa as condições de "maior estresse à rede", enquanto a Figura 1 (c) apresenta o impacto da variação da capacidade de rede. Já a Figura 1 (d) mostra o impacto das diferentes velocidades de roteador. Em todos os experimentos, o cenário 6 apresentou o melhor desempenho em relação ao tempo de execução. No "melhor cenário" - Figura 1 (a) -, por exemplo, o cenário 6 teve tempo de execução de $6321 \mathrm{~ms}$ com carga de trabalho de $1 \mathrm{~GB}$, de $83400 \mathrm{~ms}$ com $10 \mathrm{~GB}$ e de $1202534 \mathrm{~ms}$ com 100 GB. Tais resultados evidenciam a importância da transferência de dados na composição do custo total de processamento 
distribuído de hash join. O cenário 6 é o que transfere a menor quantidade de dados: apenas os dados da relação de saída de consulta. Ao mesmo tempo, possui o maior sobrecusto de armazenamento e sincronização de dados nos servidores, pois os dados estão co-localizados. O segundo melhor desempenho foi obtido com o cenário 4 , que trafega a segunda menor quantidade de dados: hash join e relação de saída. O cenário 1 deveria ser mais lento do que o 2, pois o primeiro transfere dados da relação maior e o segundo da menor. No entanto, ambos equiparam-se porque são solicitados mais dados provenientes da relação menor. O desempenho em relação ao tempo de execução com comunicação full-duplex dos cenários 3 e 5, quando com capacidade do roteador idêntica à das CPUs dos servidores, é igual ao dos cenários 1 e 2. Quando se adota a comunicação half-duplex, os cenários 1 e 2 são mais rápidos do que os cenários 3 e 5 . À medida que se aumenta a velocidade da rede, diminui-se o gap entre os pares de cenários. Quanto menor a carga de trabalho, também é menor esse gap. Por fim, um processamento feito no roteador com capacidade $5 \%$ menor afeta o tempo total dos cenários 3 e 5 em 3,25\%, enquanto um feito no roteador com capacidade $10 \%$ menor aumenta em $6,50 \%$.

\section{Conclusões e Trabalhos Futuros}

Neste artigo, foi apresentada uma análise de diferentes cenários de execução de hash join distribuído, incluindo execução da operação em um roteador, a partir de um modelo de custo. Espera-se que os resultados aqui apresentados contribuam para um conhecimento mais amplo sobre diferentes estratégias de processamento distribuído de dados e o potencial do processamento de dados em dispositivos de rede para ganho de desempenho, motivando novos estudos. Como trabalhos futuros, pretende-se analisar outras consultas que realizam a operação de hash join com o modelo aqui apresentado e criar um ambiente virtualizado para simular os diferentes cenários e as implicações do processamento do hash join distribuído no roteador, simulando a tecnologia SD-WAN.

\section{Referências}

Council, T. P. (2020). Tpc benchmark h. http://www.tpc.org/tpch/. Acessado em $25 / 11 / 2020$.

Estébanez, C., Sáez, Y., Recio, G., and Isasi, P. (2014). Performance of the most common non-cryptographic hash functions. Software: Practice and Experience, 44.

Fowler, G., Vo, P., and Noll, L. C. (2013). Fnv hash. http://www. isthe.com/ chongo/tech/comp/fnv/index.html/. Acessado em 14/04/2020.

Kossmann, D. (2000). The state of the art in distributed query processing. 32(4):422-469.

Scheidt de Cristo, F., Almeida, E., and Alves, M. (2019). Vivid cuckoo hash: Fast cuckoo table building in simd. In Simp. em Sistemas Computacionais de Alto Desempenho.

Shin, M., Nam, K., and Kim, H. (2012). Software-defined networking (sdn): A reference architecture and open apis. In Int. Conf. on ICT Convergence, pages 360-361.

Yang, Y. and Singhal, M. (1997). A comprehensive survey of join techniques in relational databases.

Yang, Z., Cui, Y., Li, B., Liu, Y., and Xu, Y. (2019). Software-defined wide area network (sd-wan): Architecture, advances and opportunities. In 2019 28th International Conference on Computer Communication and Networks (ICCCN), pages 1-9. 